\title{
Efficient polarization qubit transmission assisted by frequency degree of freedom
}

\author{
$\mathrm{Xi}-\mathrm{Han} \mathrm{Li}^{*}$ \\ Department of Physics, College of Physics, Chongqing University, Chongqing 400044, China
}

(Dated: June 7, 2018)

\begin{abstract}
We present an efficient arbitrary polarization qubit transmission scheme against channel noise by utilizing frequency degree of freedom, which is more stable in transmission surroundings. The information of quantum state is encoded in frequency state during the transmission and transferred to polarization state later. Both the fidelity of quantum state transmitted and the success probability of this scheme are 1 in principle.
\end{abstract}

PACS numbers: 03.67Pp, 03.67.Hk, 03.65.Ud

The transmission of quantum states between participates is an essential step in each quantum communication protocol. However, the fidelity of a quantum state rapidly degrades because of the photon loss and decoherence during the transmission, which will impact the efficiency and the security of quantum communication consequently. The coupling between quantum system and the environment, which is called as noise, is a serious obstacle to a perfect communication. The polarization degree of freedom (DOF) of photons are usually chosen as information carries because of its maneuverability. However, this DOF is incident to be influenced by thermal fluctuation, vibration and imperfection of fibers, i.e, the channel noise. Various methods had been proposed for rejecting or correcting the errors caused by noise, one of which can be called as quantum error rejection [1 4]

In quantum error rejection schemes, there is an important assumption that the variation of noise is slow. If several qubits transmit through the channel simultaneously or close to each other, the impact of noise is identical. With this hypothesis, arbitrary quantum state can be transmitted probabilistically by making two parts suffer from the same noise to interact and then reject errors by postselection. In 2005, Kalamidas proposed a single-photon error rejection protocol [1], which encodes the quantum states in two time-bins and an uncorrupted state can be obtained at a definite time of arrival. However, fast polarization modulator are employed in this scheme, whose synchronization makes it difficult to implement. Later, we presented a faithful qubit transmission scheme with passive linear optics [2]. Yamamoto et al proposed a qubit distribution scheme with ancillary qubit in 2005 3]. However, the success probability is a bit low. All of these three protocols utilize the temporal DOF to encode the state where losses will be introduce inevitably during the postselection to rebuild the original state.

Recently, some other DOFs besides polarization attract much attention, such as frequency, spatial mode, orbital angular momentum, transverse spatial mode, and so on. Since operations in one DOF do not perturb the

\footnotetext{
${ }^{*}$ Corresponding author: xihanli@cqu.edu.cn
}

others, photons with multi-DOF may provide novel ways to realize quantum information process. Frequency DOF has been used in a series of quantum information schemes because of its stability [4 11]. We proposed an efficient qubit transmission scheme [4] with frequency DOF whose success probability is four times of Ref. [3].

In this letter, we present a new method for transmitting a single photon polarization state against channel noise with frequency DOF. The sender encodes the information of quantum state into frequency DOF and the receiver converts the information back to the polarization DOF and obtain the uncorrupted state deterministically.

Let us suppose that an arbitrary single-photon pure state to be transmitted is written as

$$
|\psi\rangle=\alpha|H\rangle+\beta|V\rangle, \quad\left(|\alpha|^{2}+|\beta|^{2}=1\right)
$$

Here $|H\rangle$ and $|V\rangle$ represent the horizontal and the vertical polarization states of a single photon, respectively. And $\alpha$ and $\beta$ are two parameters which carry the information of the state to be transmitted. The frequency of this photon is prepared in $\omega_{2}$. Considering the two DOFs, the whole quantum state to be transmitted can be written as

$$
|\psi\rangle=\alpha\left|H, \omega_{2}\right\rangle+\beta\left|V, \omega_{2}\right\rangle
$$

Before the transmission, the sender encodes the state with the encoder composed of a polarizing beam splitter (PBS), a beam splitter (BS) and a half wave plate (HWP), shown in Fig.1. PBS transmits the horizontal polarization mode $|H\rangle$ and reflects the vertical one $|V\rangle$. In this way, the state $|H\rangle$ propagates through the path 1 and the state $|V\rangle$ goes though path 2 . The two pathes have the same length. The frequency shifter (FS) in path 1 modulates the frequency from $\omega_{2}$ to $\omega_{1}$ and the HWP in path 2 affects the polarization modes as the bit-flip operation $\sigma_{x}$, i.e., $|H\rangle \leftrightarrow|V\rangle$. 


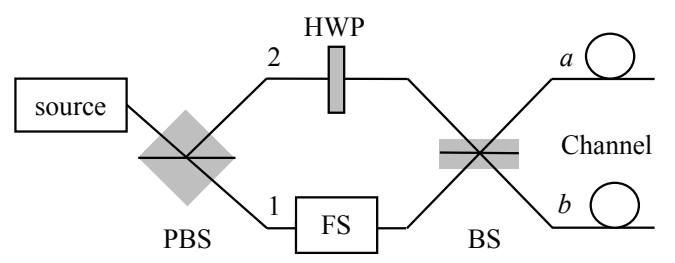

FIG. 1: The schematic diagram of the encoder. Path 1 and 2 have the same length. The Sender encodes his qubit into a frequency superposition state and transmits it to the receiver through two independent noisy channels.

The initial state evolves in the encoder as follows,

$$
\begin{array}{ll}
|\psi\rangle \stackrel{\text { ․ }}{=} & \alpha\left|H, \omega_{2}\right\rangle+\beta\left|V, \omega_{2}\right\rangle \\
\stackrel{F B S}{\longrightarrow} & \alpha\left|H, \omega_{2}\right\rangle_{1}+\beta\left|V, \omega_{2}\right\rangle_{2} \\
\stackrel{F S}{H W P} & \alpha\left|H, \omega_{1}\right\rangle_{1}+\beta\left|H, \omega_{2}\right\rangle_{2} \\
\stackrel{B S}{\longrightarrow} & \frac{1}{\sqrt{2}}\left[\left(\alpha\left|H, \omega_{1}\right\rangle+i \beta\left|H, \omega_{2}\right\rangle\right)_{a}\right. \\
& \left.+\left(i \alpha\left|H, \omega_{1}\right\rangle+\beta\left|H, \omega_{2}\right\rangle\right)_{b}\right] .
\end{array}
$$

The subscripts 1 and 2 represent the two pathes of the interferometer, and $a$ and $b$ represent the two output ports of BS and two independent noisy channels. The coefficient $i$ comes from the the phase shift aroused by the reflection of BS. From the last two lines, we find the information of the initial state is encoded into a frequency superposition state, which is immune to the channel noise. And these two states in different noise channel have similar form, we just analyze the situation of channel $a$ in detail below.

The channel noise can be expressed with a unitary transformation

$$
|H\rangle \rightarrow \delta|H\rangle+\eta|V\rangle,\left(|\delta|^{2}+|\eta|^{2}=1\right)
$$

where $\delta$ and $\eta$ are the parameters of the noise. The quantum state the receiver get from channel $a$ can be written as

$$
\begin{aligned}
& \frac{1}{\sqrt{2}}\left(\alpha\left|H, \omega_{1}\right\rangle+i \beta\left|H, \omega_{2}\right\rangle\right)_{a} \stackrel{\text { noise }}{\longrightarrow}|\psi\rangle_{a} \\
& =\frac{1}{\sqrt{2}}\left(\alpha \delta\left|H, \omega_{1}\right\rangle+\alpha \eta\left|V, \omega_{1}\right\rangle+i \beta \delta\left|H, \omega_{2}\right\rangle+i \beta \eta\left|V, \omega_{2}\right\rangle\right)
\end{aligned}
$$

The receiver uses a decoder to obtain the original polarization state, shown in Fig.2. The wavelength division multiplexer (WDW) is polarization independent and used to guide photons to different spatial modes according to their frequencies. That is, frequency state $\omega_{1}\left(\omega_{2}\right)$ goes to path 1 (2) according to the figure. The FS in path 1 adjusts the frequency state from $\omega_{1}$ to $\omega_{2}$, so as to make the quantum states in these two pathes have the same frequency. And the HWP in path 2 rotates the horizontal polarization $|H\rangle$ to $|V\rangle$. The phase modulator $(\mathrm{PM})$ modulates the phase of quantum state passing

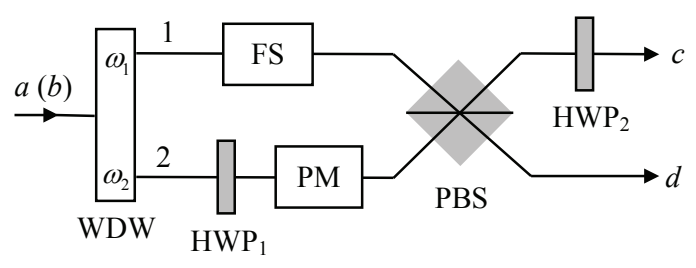

FIG. 2: The schematic diagram of the decoder. Wavelength division multiplexer (WDW) guides photon to different spatial modes according to its frequency. Half wave plates (HWP) act as a bit-flip operation and the phase modulator (PM) adjusts the phase by $\pi / 2$. The frequency shifter (FS) modulates the frequency so as to eliminate the frequency difference between different pathes.

through path 2 as $|\psi\rangle \rightarrow e^{i \varphi}|\psi\rangle$, where $\varphi=\pi / 2$. The effect of the decoder can be written as

$$
\begin{aligned}
|\psi\rangle_{a} \stackrel{W D M}{\longrightarrow} & \frac{1}{\sqrt{2}}\left(\alpha \delta\left|H, \omega_{1}\right\rangle_{1}+\alpha \eta\left|V, \omega_{1}\right\rangle_{1}\right. \\
& \left.+i \beta \delta\left|H, \omega_{2}\right\rangle_{2}+i \beta \eta\left|V, \omega_{2}\right\rangle_{2}\right) \\
\stackrel{F S, P M}{H W P_{1}} & \frac{1}{\sqrt{2}}\left(\alpha \delta\left|H, \omega_{2}\right\rangle_{1}+\alpha \eta\left|V, \omega_{2}\right\rangle_{1}\right. \\
& \left.+\beta \delta\left|V, \omega_{2}\right\rangle_{2}+\beta \eta\left|H, \omega_{2}\right\rangle_{2}\right) \\
\stackrel{P B S}{\longrightarrow W P_{2}} & \frac{1}{\sqrt{2}}\left[\delta\left(\alpha\left|H, \omega_{2}\right\rangle+\beta\left|V, \omega_{2}\right\rangle\right)_{d}\right. \\
& \left.+\eta\left(\alpha\left|H, \omega_{2}\right\rangle+\beta\left|V, \omega_{2}\right\rangle\right)_{c}\right] .
\end{aligned}
$$

The subscripts 1 and 2 represent two pathes and $c, d$ represent two output ports of the decoder. These two states in two different spatial modes have the same form with the initial one, retaining the information of the quantum state. With the decoder set in channel $a$, the receiver can get the initial state with success probability $1 / 2$. The situation of channel $b$ is similar. Suppose the noise is $|H\rangle \rightarrow \delta^{\prime}|H\rangle+\eta^{\prime}|V\rangle$ in channel $b$, the state received with another decoder set in channel $b$ is

$$
\begin{aligned}
|\psi\rangle_{b} \stackrel{\text { decoder }}{\longrightarrow} & \frac{i}{\sqrt{2}}\left[\delta^{\prime}\left(\alpha\left|H, \omega_{2}\right\rangle+\beta\left|V, \omega_{2}\right\rangle\right)_{d}\right. \\
& \left.+\eta^{\prime}\left(\alpha\left|H, \omega_{2}\right\rangle+\beta\left|V, \omega_{2}\right\rangle\right)_{c}\right],
\end{aligned}
$$

which are also equal states with the original one. Therefore the total success probability to transmit an arbitrary polarization state is 1 in principle.

In the present scheme, the frequency DOF is introduced to defeat the channel noise. Before the transmission, the secret information encoded in polarization state was transferred to frequency DOF, which will be converted back to original polarization state after transmission. Therefore the modulation of frequency DOF is important in our protocol. The FS, which can be carried out by acousto-optic modulator (AOM) [12, 13] or sum-frequency generation process [14], is used to shift the frequency state of photons. And the WDM works as a frequency beam splitter [9, 10], which guides photons 
with different frequency to different spatial modes, can also be substituted by a fiber bragg grating (FBG) [7, 8]. In a word, the operations required by our scheme can be implemented with current technology.

In our scheme, two decoders are used because the quantum state transmits through two independent noisy channels. In order to save costs, only one decoder is required with the upgrade of the encoder, as shown in Fig.3. The two states exit from two output ports of BS are guided to the same noisy channel by means of an unbalance interferometer. Pathes 3 and 4 have different length and a state passing through path 3 will be delay by $\Delta T$. Then the state launched into the noisy channel can be

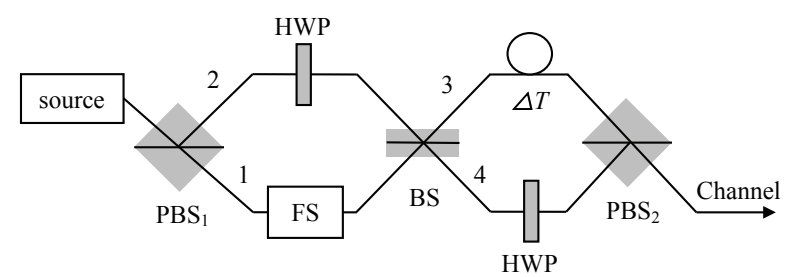

FIG. 3: The schematic diagram of the upgraded encoder. The encoded quantum state is imported into one noisy channel by means of an unbalance interferometer. During the transmission, the superposition of state is both in time and frequency.

described as

$$
\begin{aligned}
& \frac{1}{\sqrt{2}}\left[\left(\alpha\left|H, \omega_{1}\right\rangle+i \beta\left|H, \omega_{2}\right\rangle\right)_{t+\Delta T}\right. \\
& \left.\quad+\left(i \alpha\left|V, \omega_{1}\right\rangle+\beta\left|V, \omega_{2}\right\rangle\right)_{t}\right] .
\end{aligned}
$$

Here $t$ and $t+\Delta T$ denotes the arrival time, where $t$ is the arrival time of the state which did not be delayed. Suppose the channel noise acts as

$$
|H\rangle \rightarrow \delta_{1}|H\rangle+\eta_{1}|V\rangle, \quad|V\rangle \rightarrow \delta_{2}|H\rangle+\eta_{2}|V\rangle .
$$

Then state after the decoder becomes

$$
\begin{gathered}
\frac{1}{\sqrt{2}}\left[\delta_{1}\left(\alpha\left|H, \omega_{2}\right\rangle+\beta\left|V, \omega_{2}\right\rangle\right)_{d(t+\Delta T)}\right. \\
\left.+\eta_{1}\left(\alpha\left|H, \omega_{2}\right\rangle+\beta\left|V, \omega_{2}\right\rangle\right)_{c(t+\Delta T)}\right] \\
+\frac{i}{\sqrt{2}}\left[\delta_{2}\left(\alpha\left|H, \omega_{2}\right\rangle+\beta\left|V, \omega_{2}\right\rangle\right)_{d(t)}\right. \\
\left.\quad+\eta_{2}\left(\alpha\left|H, \omega_{2}\right\rangle+\beta\left|V, \omega_{2}\right\rangle\right)_{c(t)}\right] .
\end{gathered}
$$

From the expression we can find that the receiver can obtain the quantum state in two different spatial modes $c$ and $d$ and in two time slots $t+\Delta T$ and $t$. However, whenever and wherever the quantum state arrives, it is always entirely consistent with the original one. It means a perfect transmission of an initially arbitrary polarization state through a noisy channel.

This scheme proposed an useful method to transmit polarization qubit against channel noise. Then it will have good applications in one way quantum communication protocols such as BB84 QKD protocol 15]. The two parties can choose the two nonorthogonal bases, $| \pm x\rangle=\frac{1}{\sqrt{2}}(|H\rangle \pm|V\rangle)$ and $| \pm y\rangle=\frac{1}{\sqrt{2}}(|H\rangle \pm i|V\rangle)$, to realize the sharing of secret keys. With our encoder and decoder, the efficiency of quantum key distribution scheme is the same as the original one and the errors caused by the channel noise are eliminated.

In summary, we have proposed a single-photon error correction scheme against collective noise with frequency DOF. In this scheme, additional qubits is not required. The polarization state is encoded in frequency DOF which is immune to noise and decrypted after transmission. The success probability is $100 \%$ in principle. As in our scheme two pulses traveling in different time slots are not interacted to get the uncorrupted state, the channel noise should not be restricted as collective one. In addition, our scheme is not only suitable for pure states but also for mixed ones, which will have good applications in one-way quantum communication.

This work is supported by the Fundamental Research Funds for the Central Universities Project under Grant No. CDJZR10100018.
[1] D. Kalamidas, Phys. Lett. A 343, 331-335 (2005).

[2] X.H. Li, F.G. Deng, H.Y. Zhou, Appl. Phys. Lett. 91, 144101 (2007).

[3] T. Yamamoto, J. Shimamura, S.K. Özdemir, M. Koashi, N. Imoto, Phys. Rev. Lett. 95, 040503 (2005).

[4] X.H. Li, B.K. Zhao, Y.B. Sheng, F.G. Deng, H.Y. Zhou, Opt. Commun. 282, 4025-4027 (2009).
[5] B.S. Shi, Y.K. Jiang, G.C. Guo, Appl. Phys. B 70, 415417 (2000).

[6] J.-M. Merolla, L. Duraffourg, J.-P. Goedgebuer, A. Soujaeff, F. Patois, W.T. Rhodes, Eur. Phys. J. D 18, 141146 (2002).

[7] M. Bloch, S.W. McLaughlin, J.-M. Merolla, F. Patois, Opt. Lett. 32 301-303 (2007). 
[8] T. Zhang, Z.Q. Yin, Z.F. Han, G.C. Guo, Opt. Commu. 281, 4800-4802 (2008).

[9] Y.B. Sheng, F.G. Deng, Phys. Rew. A 81, 042332 (2010).

[10] L. Xiao, C. Wang, W. Zhang, Y.D. Huang, J.D. Peng, G.L. Long, Phys. Rev. A 77 (2008) 042315.

[11] Y.B. Sheng, F.G. Deng, Phys. Rew. A 81, 032307 (2010).

[12] E. H. Huntington and T. C. Ralph, Phys. Rev. A 69, 042318 (2004) .

[13] E. H. Huntington, G. N. Milford, C. Robilliard, T. C.
Ralph, Opt. Lett. 30, 2481-2483 (2005).

[14] C. Langrock, E. Diamanti, R.V. Roussev, Y. Yamamoto, M.M. Fejer, Opt. Lett. 30, 1725-1727 (2005).

[15] C. H. Bennett and G. Brassard, in: Proceedings of IEEE International Conference on Computers, Systems and Signal Processing, Bangalore, India, IEEE, New York, 1984 p. 175. 